Baltic Astronomy, vol. 7, 495-512, 1998.

\title{
CIRCUMSTELLAR SHELLS OF THE MASS-LOSING AGB STARS: TERMINAL OUTFLOW VELOCITIES
}

\author{
A. Kučinskas \\ Institute of Theoretical Physics and Astronomy, Goštauto 12, \\ Vilnius 2600, Lithuania \\ National Astronomical Observatory, Osawa, Mitaka, Tokyo 181, Japan
}

Received February 23, 1998, revised August 19, 1998.

\begin{abstract}
The influence of stellar and dust-related parameters on the terminal outflow velocities in the winds of oxygen-rich and carbon-rich AGB stars is investigated. A comparison of the theoretically predicted and observed terminal outflow velocities reveals that differences in dust-to-gas ratios may account for a wide range of observed terminal gas outflow velocities and mass-loss rates on the AGB. The terminal outflow velocity can be influenced significantly by stellar luminosity and only marginally by mass, effective temperature, the average dust grain size and the position of a dustforming region in the outflow. It is evident from the model calculations that radiation pressure on dust grains may drive the outflows only in cool $\left(T_{\mathrm{e}}<2700 \mathrm{~K}\right)$, luminous $\left(L_{\star}>7 \times 10^{3} L_{\odot}\right)$ and low mass $\left(M_{\star}<3 M_{\odot}\right)$ stars. Calculations indicate that radiation pressure on dust grains generally cannot produce slow winds $(<10 \mathrm{~km} / \mathrm{s})$ at the low mass-loss rates $\left(<10^{-6} M_{\odot} /\right.$ yr $)$.
\end{abstract}

Key words: stars: asymptotic-branch, mass-loss, late-type, oxygenrich, carbon-rich

\section{INTRODUCTION}

It is widely accepted that radiation pressure on dust grains is responsible for driving winds from AGB stars. Once the temperature in the extended atmosphere drops down to $900-1400 \mathrm{~K}$, dust formation can start, if gas density is sufficient for dust growth to occur. The momentum absorbed by the newly formed dust grains 
from the stellar radiation field can be efficiently transferred to the gaseous component of the wind by collisions, producing mass-loss of the order of $10^{-7}-10^{-5} M_{\odot} / \mathrm{yr}$ (Habing 1996).

One of the parameters, which characterizes circumstellar outflows from evolved stars and which can be obtained from observations, is the terminal gas outflow velocity, $v_{\exp }$. Previous investigations indicated that numerical value of $v_{\exp }$ can be determined from various stellar and dust-related parameters, such as luminosities, dust-to-gas ratios and dust grain sizes (Netzer \& Elitzur 1993, hereafter NE; Habing et al. 1994, hereafter HTT; Kruger et al. 1994, hereafter KGS).

In this work a model of stationary dust-driven outflow is constructed, which allows to calculate terminal gas outflow velocity, $v_{\exp }$, as an eigenvalue of the model input parameters. The model predictions are used to investigate to what extent the terminal gas outflow velocity may be affected by stellar and dust parameters, tackling a question whether the observed spread in terminal outflow velocities and mass-loss rates can be produced by differences in stellar parameters and dust properties of the mass-losing stars on the AGB.

\section{THE MODEL}

\subsection{Basic equations}

A stationary spherically symmetrical outflow at a constant gas mass loss rate is assumed. Integration of the gas equation of continuity yields

$$
\dot{M}_{\mathrm{g}}=4 \pi \rho v r^{2}
$$

where $\dot{M}_{\mathrm{g}}$ is the gas mass-loss rate, $\rho$ and $v$ are the gas density and the expansion velocity and $r$ is the radial distance.

The equation of motion of the gas is

$$
v \frac{d v}{d r}=-\frac{1}{\rho} \frac{d P}{d r}-\frac{G M_{\star}}{r^{2}}+F_{\mathrm{rad}}
$$

where $P$ and $M_{\star}$ denote the gas pressure and stellar mass, $G$ is the gravitational constant and $F_{\mathrm{rad}}$ is a radiation pressure force per unit mass expelled on the dust grains. Denoting the radius of a spherical dust grain by $a_{\mathrm{d}}$, the density of the grain material by $\rho_{\mathrm{s}}$ and the dust velocity by $v_{\mathbf{d}}, F_{\text {rad }}$ is given by 


$$
F_{\mathrm{rad}}=\frac{3 L_{\star} Q_{\mathrm{pr}}}{16 c \pi \rho_{\mathrm{s}} a_{\mathrm{d}}} \frac{\dot{M}_{\mathrm{d}}}{\dot{M}_{\mathrm{g}}} \frac{v}{v_{\mathrm{d}}} \frac{1}{r^{2}},
$$

here $L_{\star}$ is the luminosity of the star, $\dot{M}_{\mathrm{d}}$ denotes dust mass-loss rate $\left(\dot{M}_{\mathrm{d}} / \dot{M}_{\mathrm{g}}\right.$ is the dust-to-gas ratio), $c$ is the speed of light in the vacuum and $Q_{\mathrm{pr}}$ is the Planck mean radiation pressure efficiency factor (e.g., Gilman 1974). Planck mean radiation pressure efficiency factors were calculated by Raudeliūnas (1997) for both silicate and amorphous carbon grains, using the optical constants of dirty silicates published by Draine (1985) and those of amorphous carbon published by Rouleau \& Martin (1991).

Detailed calculations of the carbon dust formation in the circumstellar environments of carbon-rich stars have demonstrated that the amount of dust formed in the outflow is determined by physical conditions in the dust growth region. The dust-to-gas ratio, however, rapidly reaches its terminal value, typically, within a few stellar radii (e.g., KGS). Therefore it is assumed that the radial dependence of the dust-to-gas ratio $\dot{M}_{\mathrm{d}} / \dot{M}_{\mathrm{g}}$ can be described by the following expression (since $\dot{M}_{\mathrm{d}} \ll \dot{M}_{\mathrm{g}}$, the radial variation of $\dot{M}_{\mathrm{g}}$ is neglected)

$$
\frac{\dot{M}_{d}}{\dot{M}_{g}}=\left(\frac{\dot{M}_{d}}{\dot{M}_{g}}\right)_{\infty}\left(1+\exp \frac{T-T_{c}}{\Delta T}\right)^{-1}
$$

where $\left(\dot{M}_{\mathrm{d}} / \dot{M}_{\mathrm{g}}\right)_{\infty}$ is the final dust-to-gas ratio, $T$ is gas temperature (see equation (7) below), $T_{\mathrm{c}}$ is the dust condensation temperature and $\Delta T$ is the width of the dust condensation zone.

While the validity of the complete momentum coupling between dust and gas has been questioned in numerous studies (Berruyer \& Frisch 1983; MacGregor \& Stencel 1992; Hartquist \& Havnes 1994; HTT; Mastrodemos et al. 1996), it is widely accepted that it holds for a wide interval of the outflow velocities and densities. Thus, in this work we assume that the dust is fully momentum coupled to the gas by collisions. Dust velocity then is given by

$$
v_{\mathrm{d}}=v+\left(\frac{Q_{\mathrm{pr}} L_{\star} v}{\dot{M}_{\mathrm{g}} c}\right)^{1 / 2} .
$$

Assuming that gas is ideal, $P=\rho / \mu R T$, where $\mu$ is the mean molecular weight and $R$ is the universal gas constant. Combining 
equations (1), (2) and taking into account the equation of state for ideal gas, the equation of gas motion may be written in the following form:

$$
\frac{d v}{d r}=\frac{v}{v^{2}-u^{2}}\left(\frac{2 u^{2}}{r}-\frac{R}{\mu} \frac{d T}{d r}-\frac{G M_{\star}}{r^{2}}+F_{\mathrm{rad}}\right) .
$$

Equation (6) has a singular point at $r=r_{\mathbf{s}}$, where $v\left(r_{\mathbf{s}}\right)=u\left(r_{\mathbf{s}}\right)$, here $u$ is the sound velocity, defined as $u^{2}=R / \mu T$. A special care has to be taken to maintain a regular solution at this point (see below).

KGS have demonstrated that a proper treatment of the gas energy balance equation may lead to the gas temperatures which are higher by up to several hundred degrees than the gas equilibrium temperatures. This effect, however, proved to have a minor importance on the dynamical structure of the wind. Furthermore, various power law approximations of a radial temperature dependence have been used successfully to model the structure of the circumstellar shells (Gail \& Sedlmayr 1987; MacGregor \& Stencel 1992; Egan \& Leung 1995), giving a good agreement with the observable quantities, such as mass-loss rate and terminal gas outflow velocity. Thus, instead of solving the gas energy balance equation, we assume that the gas temperature is given by a simple power law

$$
T=T_{\mathrm{e}}\left(\frac{R_{\star}}{r}\right)^{\beta},
$$

where $T_{\mathrm{e}}$ is the effective temperature, $R_{\star}$ is the stellar radius and the typical value of $\beta$ is between 0.4 and 0.8 (Papoular \& Pegourie 1986, Egan \& Leung 1995).

We approximate the growth of the mean radius of a dust grain by the following expression

$$
a_{\mathrm{d}}=a_{0}+\frac{a_{\infty}}{1+\exp \frac{T-T_{c}}{\Delta T}},
$$

where $a_{0}$ and $a_{\infty}$ denote initial and final grain radii, respectively, $T_{\mathrm{c}}$ is the dust condensation temperature and $\Delta T$ is the width of the dust condensation zone. The choice of expression (8) is based on theoretical models of the amorphous carbon grain growth in the circumstellar shells of carbon-rich stars (Gail \& Sedlmayr 1987, HTT, Bowen 1988). This expression approximates the growth of the grain radius in a simple way, retaining a reasonable accuracy with respect 
to the original modeling results. In this work (8) is used for both silicate and carbonaceous dust grains.

It has to be noted, that assumption of the unique dust grain size sets additional limitations to the model. Indeed, in reality a grain-size distribution has to be assumed instead of a single $a_{\mathrm{d}}$. Jura (1994) has demonstrated, that in order to explain observations, two grain sizes were needed to be present in the outer shell of the famous carbon-rich star IRC+10216: small grains, to shield the molecules in the circumstellar shell from the destruction by interstellar ultraviolet radiation, and large grains, to produce circumstellar polarization in the $K$ band. Theoretical investigations of evolution of the grain size distribution in one-dimensional stationary flows (Dominik et al. 1989; Kruger et al. 1995; Kruger \& Sedlmayr 1997, hereafter KS) have demonstrated the importance of the grain size distribution related effects in the winds from carbon-rich stars. However, the detailed investigation of this problem is beyond the scope of this work.

\subsection{Method of solution}

The integration procedure of the flow equations is similar to that proposed by Gail \& Sedlmayr (1985). The free parameters to be specified at the beginning of the integration are: $M_{\star}, L_{\star}, T_{e}, T_{\mathrm{c}}$, $\dot{M}_{\mathrm{g}}, a_{\mathrm{d}}$ and $\dot{M}_{\mathrm{d}} / \dot{M}_{\mathrm{g}}$ (the latter two given by $a_{\infty}$ and $\left(\dot{M}_{\mathrm{d}} / \dot{M}_{\mathrm{g}}\right)_{\infty}$, respectively). The initial gas velocity $v_{0}\left(R_{\star}\right)$ is chosen by trial and the equation of motion is integrated outward from the stellar surface, which is defined as

$$
R_{\star}=\left(\frac{L_{\star}}{4 \pi \sigma T_{\mathrm{e}}^{4}}\right)^{1 / 2}
$$

Let us denote the sonic point by setting $r=r_{\mathrm{s}}$. The regularity condition at the sonic point is

$$
\left(\frac{2 u^{2}}{r}-\frac{R}{\mu} \frac{d T}{d r}-\frac{G M_{\star}}{r^{2}}+F_{\mathrm{rad}}\right) \mid r=r_{s}=0 .
$$

The initial gas velocity at the stellar surface, $v_{0}\left(R_{\star}\right)$, is varied by an iterative procedure until the condition (10) is satisfied and the wind velocity makes a smooth transition from the subsonic into the supersonic regime. Therefore, the regularity condition (10) sets the requirement on the uniqueness of the wind velocity profile. 
Integration of the flow equations is started at the stellar surface and followed through a smooth transition of the sonic point up to a selected outer radius $r=R_{\infty}$. After the wind velocity profile is obtained, expression (1) may be used to estimate the gas density on the stellar surface, since the mass loss rate is set as a model parameter prior to calculation. Generally, gas densities in the upper layers of the extended atmospheres of the evolved giants and supergiants are poorly known, and they may be influenced by various factors, such as gas opacities (Salaris \& Cassisi 1996), effects of spherical extension (Jorgensen et al. 1992), extension of the photospheric layers because of radiation pressure on molecules (Jorgensen \& Johnson 1992; Jorgensen 1994), rotation, stellar pulsations and/or shock waves. However, certain limiting values can be set, being based, for instance, on calculations of the theoretical model atmospheres of evolved stars (Plez 1992). Such a condition may be written as

$$
\rho_{\min } \leq \rho\left(R_{\star}\right) \leq \rho_{\max } .
$$

Therefore, each model run is also a subject to the condition (11). If this condition is not satisfied, the integration procedure is repeated with the new guess of $\dot{M}_{\mathrm{g}}$ and is finally stopped when both conditions (10) and (11) are satisfied. Changing the mass-loss rate it is possible to obtain a set of different models for the same set of model input parameters, keeping the gas density on the stellar surface within the limits set by (11). Indeed, if the gas density on the stellar surface is specified as a single value, calculations will yield the mass-loss rate as an eigenvalue of a specified model parameter set.

\subsection{Comparison with the existing wind models}

Ideally, the only free parameters of any theoretical wind model should be those describing a star itself, such as its mass, age, chemical composition etc. Indeed, stellar parameters govern the evolutionary path of any particular star on the AGB and beyond, and they are tightly interrelated. Therefore, the occurrence of wind from a particular star on the AGB and the wind parameters might be determined by its evolutionary status, producing a single-valued wind solution for any star on the HR diagram.

In reality, however, many simplifying assumptions about various physical processes, as well as the geometry of the outflow have to be made, which limit a self-consistency of the model considerably. 
Our model suffers from several limitations too. The major ones are: assumptions of the stability and spherical symmetry of the wind, simplified treatment of the dust formation process, neglected influence of the gas energy balance and energy exchange between the dust and gas.

However, a significant amount of the spectral energy distribution curves of the AGB stars can be successfully interpreted within a framework of a stationary spherically symmetrical wind model, assuming even the instant dust formation and simplified treatment of the radiative transfer problem (e.g., Ivezic \& Elitzur 1995, 1996; Groenewegen 1997). This suggests, that even such simplified models can be used successfully to investigate certain properties of the mass-losing stars on the AGB.

The comparison of our model with the models of other authors is not straightforward, primarily because of a different model assumptions. For instance, the gas pressure is neglected in the models of NE and HTT. However, as it has been demonstrated by Steffen et al. (1997, hereafter SSMS), the gas pressure plays an important role in the wind dynamics, and may stipulate an increase in the terminal gas outflow velocities by up to $40 \%$ with respect to the models where the gas pressure is neglected. It has been also indicated by SSMS that the gas pressure is particularly important in winds with low terminal outflow velocities $\left(v_{\exp }<30 \mathrm{~km} / \mathrm{s}\right)$.

We have calculated several models, for which model input parameters where chosen to match exactly the parameters used by SSMS in their hydrodynamic models of carbon-rich stars. The calculated terminal outflow velocities are plotted versus the mass-loss rates in Fig. 1. For the comparison, the terminal outflow velocities calculated by SSMS are also plotted. Although terminal outflow velocities produced by our model are systematically higher by 2$3 \mathrm{~km} / \mathrm{s}$, the general agreement between both model results is very good, even if the radiation transfer is not treated explicitly in our model.

\section{ASTROPHYSICAL DATA FOR THE COMPARATIVE ANALYSIS}

The model employed in this work is defined by a number of input parameters. In order to compare predicted and observed $v_{\exp }$ and to investigate the influence of the involved stellar and dust parameters on the dynamics of the outflow, we calculate the terminal gas outflow 

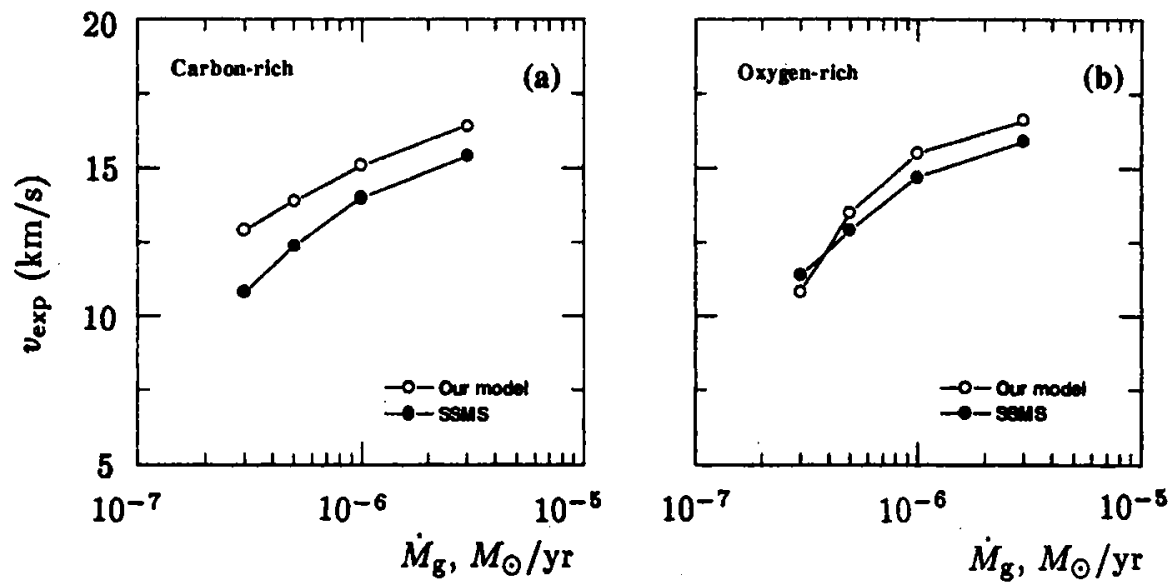

Fig. 1. A comparison of the terminal outflow velocities produced by our model and the model of SSMS for carbon-rich (a) and oxygen-rich (b) AGB stars: (a) model parameters: $M_{\star}=1 M_{\odot}, L_{\star}=10^{4} L_{\odot}, T_{\mathrm{e}}=$ $2000 \mathrm{~K}, a_{\mathrm{d}}=0.05 \mu \mathrm{m}, \rho_{\mathrm{s}}=1.85 \mathrm{~g} / \mathrm{cm}^{3}, \dot{M}_{\mathrm{d}} / \dot{M}_{\mathrm{g}}=0.0015$ and $T_{\mathrm{c}}=850 \mathrm{~K}$ (amorphous carbon grains are assumed); (b) model parameters: $M_{\star}=1$ $M_{\odot}, L_{\star}=10^{4} L_{\odot}, T_{\mathrm{e}}=3500 \mathrm{~K}, a_{\mathrm{d}}=0.05 \mu \mathrm{m}, \rho_{\mathrm{s}}=3.28 \mathrm{~g} / \mathrm{cm}^{3}, \dot{M}_{\mathrm{d}} / \dot{M}_{\mathrm{g}}$ $=0.005$ and $T_{\mathrm{c}}=850 \mathrm{~K}$ (silicate grains are assumed). See the text for discussion.

Table 1. Parameters of the standard model.

\begin{tabular}{llll}
\hline Parameter & Oxygen-rich & & Carbon-rich \\
\hline$M_{\star}$ & & $1 M_{\odot}$ & \\
$L_{\star}$ & & $10^{4} L_{\odot}$ & \\
$T_{\mathrm{e}}$ & & $2000 \mathrm{~K}$ & \\
$\dot{M}_{\mathrm{d}} / \dot{M}_{\mathrm{g}}$ & 0.006 & & 0.002 \\
$a_{\mathrm{d}}$ & $0.05 \mu \mathrm{m}$ & & $0.03 \mu \mathrm{m}$ \\
$\rho_{\mathrm{s}}$ & $3.28 \mathrm{~g} / \mathrm{cm}^{3}$ & & $2.00 \mathrm{~g} / \mathrm{cm}^{3}$ \\
$T_{\mathrm{c}}$ & & $1100 \mathrm{~K}$ & \\
$M_{\mathrm{g}}$ & & $3 \times 10^{-6} M_{\odot} / \mathrm{yr}$ & \\
\hline
\end{tabular}

velocities for a set of different stellar models, covering a range of the observed mass-loss rates, stellar and dust-related parameters. The model parameters used in our calculations are discussed below. Parameters of the standard model are summarized in Table 1. 


\subsection{Stellar masses}

The masses of the AGB stars are so far poorly known. Recent theoretical investigations demonstrate that stars with ZAMS masses of $M_{\star} \leq 5-7 M_{\odot}$ will probably end up their AGB evolution as white dwarfs (Blocker 1995, Marigo et al. 1996). The masses of white dwarfs span quite a narrow interval with a strong peak around $M_{\mathrm{wd}} \approx 0.55-0.60 M_{\odot}$ (Weidemann 1987 ), which sets a limit on the low-mass end on the AGB. Since the intense mass-loss in previous evolutionary stages can lower the high-mass limit, the models with masses within a range of $0.7 M_{\odot} \leq M_{\star} \leq 5 M_{\odot}$ are studied in the present work.

\subsection{Stellar effective temperatures and luminosities}

Stellar luminosities on the AGB are generally in the range of $\left(3 \times 10^{3}-3 \times 10^{4}\right) L_{\odot}$, although slightly different values are quoted for oxygen-rich and carbon-rich stars. The mean luminosity for the galactic oxygen-rich AGB stars is often assumed to be equal to $L_{\star} \approx$ $5000 L_{\odot}$, while for carbon-rich stars a slightly higher value of $L_{\star} \approx$ $7000 L_{\odot}$ is quoted (e.g., Groenewegen et al. 1995). We assume an identical luminosity range of $\left(3 \times 10^{3}-3 \times 10^{4}\right) L_{\odot}$ for both oxygenrich and carbon-rich stars.

There is much ambiguity with respect to the effective temperatures on the AGB. Since the majority of stars on the AGB are enshrouded in circumstellar shells, precise determination of their effective temperatures becomes rather complicated. A sample of galactic carbon stars studied by Olofsson et al. (1993b) yields effective temperatures in a range of 2400-3200 K, with a median of $T_{\mathrm{e}}=$ $2700 \mathrm{~K}$. However, as was noted by Dominik et al. (1990), the temperature being used as a model parameter for the calculations of dust driven winds may be lower than the temperature determined by various observational methods. For this work a temperature range of $1800-3300 \mathrm{~K}$ has been chosen.

\subsection{Dust parameters}

During the recent years a considerable understanding has been reached about dust formation processes both in oxygen-rich and carbon-rich environments (Beck et al. 1992, Fleischer et al. 1992, Dominik et al. 1993, Cadwell et al. 1994, Egan \& Leung 1995, Kohler 
et al. 1997; for earlier studies refer to Gail 1992 for a review). It is widely accepted that amorphous carbon (rather than graphite) grains are formed in the outflows from carbon-rich stars. Classical nucleation theory yields dust condensation temperatures within a range of 1200-1400 K (KGS; KS). A chemical nucleation pathway approach of carbon soot formation indicates somewhat lower dust condensation temperatures, ranging between $800 \mathrm{~K}$ and $1100 \mathrm{~K}$ (Cherchneff, Barker \& Tielens 1992). It is frequently noted that polycyclic aromatic hydrocarbon (PAH) molecules might be also abundant in the circumstellar outflows from carbon-rich stars (Allain et al. 1997), although dynamically their influence was found to be of minor importance (Cherchneff, Barker \& Tielens 1991, Helling et al. 1996).

Rowan-Robinson \& Harris $(1982,1983)$ have successfully fitted the spectral energy distributions of the oxygen-rich AGB star sample using two dust condensation temperatures: $T_{\mathrm{c} 1}=1000 \mathrm{~K}$ and $T_{\mathrm{c} 2}=500 \mathrm{~K}$. Theoretical models of dust formation in the outflows of M-type stars calculated by Gail \& Sedlmayr (1986) have demonstrated that these temperatures may correspond to the condensation temperatures of $\mathrm{MgO}$ and $\mathrm{SiO}$, respectively. Tielens (1990) has presented a scenario in which silicate-rich grains may form at temperatures ranging between $1600 \mathrm{~K}$ and $1000 \mathrm{~K}$. Though the conditions in circumstellar outflows may not be favorable for all compounds to form, it is clear, that a heterogeneous dust formation is very likely to take place in the circumstellar shells of oxygen-rich stars (Dominik et al. 1993). This may lead to a much more extended dust condensation zone, allowing for the growth of more complex dust grains.

In view of these facts we chose an identical dust formation temperature range of 900-1400 $\mathrm{K}$ for both oxygen-rich and carbon-rich environments. The width of the dust condensation region is set to $\Delta T=40 \mathrm{~K}$. The initial mean grain radius is assumed to be $a_{0}=1.28 \times 10^{-4} \mu \mathrm{m}$ (Gail et al. 1984). We assume the final mean grain radii are in a range of $0.005-0.2 \mu \mathrm{m}$ for both silicate and amorphous carbon grains.

\subsection{Temperature law}

According to model calculations used to fit the spectral energy distributions of AGB stars, the decline of the gas temperature in the inner parts of the shell was frequently found to be steeper than the power law with an exponent $\beta=0.5$ (e.g., Rowan-Robinson \& 
Harris 1983, Groenewegen 1997), retaining much shallower radial temperature dependence in the outer regions $(\beta \approx 0.4)$. Similar radial temperature dependence was produced by the theoretical models of KGS, which involved a detailed gas energy balance treatment. Egan \& Leung (1995) have fitted the temperature stratification in the circumstellar outflow obtained by the radiative transfer model calculations with $\beta=0.6$. Since most of the acceleration is attained by the wind in the vicinity of the sonic point, it is essential to have an accurate temperature law in the inner part of the shell. Thus, in our work the exponent of the temperature law is chosen to be equal to $\beta=0.6$.

\subsection{Dust-to-gas ratios}

In this work the following range of dust-to-gas ratios was studied: $0.0005<\left(\dot{M}_{\mathrm{d}} / \dot{M}_{\mathrm{g}}\right)_{\infty}<0.009$. As typical values, the dust-to-gas ratios $\left(\dot{M}_{\mathrm{d}} / \dot{M}_{\mathrm{g}}\right)_{\infty}=0.006$ and 0.002 were chosen for oxygen-rich and carbon-rich stars, respectively. While the value for oxygen-rich stars is in good agreement with observations (e.g., Ivezic \& Elitzur 1995), the value assumed for carbon-rich stars is by a factor of 1.5 lower than the average value obtained from observations of Olofsson et al. (1993a). This problem will be discussed further in Section 5.

\subsection{Terminal outflow velocities}

Integration of the flow equations was started at the stellar surface and stopped at the outer boundary $R_{\infty}$. The terminal outflow velocity was then calculated as $v_{\exp }=v\left(R_{\infty}\right)$. The outer integration boundary in our calculations was set to $R_{\infty}=1000 R_{\star}$.

\subsection{Stellar sample}

The stellar sample used for the comparison of the model calculations and observations is taken from Loup et al. (1993). This sample contains terminal outflow velocities and mass-loss rates for 184 oxygen-rich and 205 carbon-rich stars, determined from the observations of ${ }^{12} \mathrm{CO}(1-0)$ and ${ }^{12} \mathrm{CO}(2-1)$. 


\section{DYNAMICS OF THE WIND: $v_{\exp }-\dot{M}_{\mathrm{g}}$ PLANE}

The strong dependence of the terminal wind velocities on the amount of dust available in the outflow was first indicated by theoretical models of HTT. This trend is also confirmed by our calculations (Fig. 2). Differences in the dust-to-gas ratios can account for the whole range of the observed $v_{\text {exp }}$ and mass-loss rates for both oxygenrich and carbon-rich AGB stars. The only exceptions are slow outflows $\left(v_{\exp }<10 \mathrm{~km} / \mathrm{s}\right)$ at low mass-loss rates $\left(\dot{M}_{\mathrm{g}}<10^{-6} M_{\odot} / \mathrm{yr}\right)$. In order to match the range of the observed $v_{\exp }$ in carbon-rich stars, we have lowered the amount of dust available in the shells of these stars down to $\dot{M}_{\mathrm{d}} / \dot{M}_{\mathrm{g}}=0.002$. Although some carbon stars may indeed possess low dust-to-gas ratios (Olofsson et al. 1993a), this value is generally lower than the usually quoted average for carbonrich stars, which is $\dot{M}_{\mathrm{d}} / \dot{M}_{\mathrm{g}} \geq 0.003$. It is important to note the following. Ideally, $Q_{\mathrm{pr}}$ used in our calculations has to be replaced by the radiation pressure factor averaged over the local radiation field of the outflow. Such approach would require a solution of the radiation transfer problem which is beyond the scope of this study. As it was shown by HTT, in such a case the resulting $Q_{\mathrm{pr}}$ will be dependent on the radial distance not only because of different grain radii, but also because of different local radiation field, and generally will be lower than $Q_{\mathrm{pr}}$ used in our work. Thus, the requirement for the lower dust-to-gas ratios may emanate from the fact that in our work $Q_{\mathrm{pr}}$ was overestimated in the dynamically important regions of the outflow.

Fig. 2 allows to conclude that the grains formed in the winds from AGB stars are smaller than $a_{\mathrm{d}}<0.2 \mu \mathrm{m}$. This is in agreement both with the dust grain sizes derived from observations (Martin \& Rogers 1987, Seab \& Snow 1989, Bagnulo et al. 1995) and theoretical predictions (NE, KGS, KS). The spread of the resulting terminal gas outflow velocities, because of the different grain radii, is quite large too. The resulting terminal outflow velocities are generally always larger than $10-15 \mathrm{~km} / \mathrm{s}$.

The dependence of the terminal outflow velocities on the grain size is quite complicated, although qualitatively similar for both oxygen-rich and carbon-rich stars. For small grain sizes up to $a_{\mathrm{d}}<0.1 \mu \mathrm{m}$ terminal outflow velocity is almost independent of grain size. Further on, $v_{\exp }$ starts to increase with increasing $a_{\mathrm{d}}$ and reaches a maximum near $a_{\mathrm{d}} \approx 0.2-0.4 \mu \mathrm{m}$, while for the larger grains terminal outflow velocity decreases again. This non-trivial 

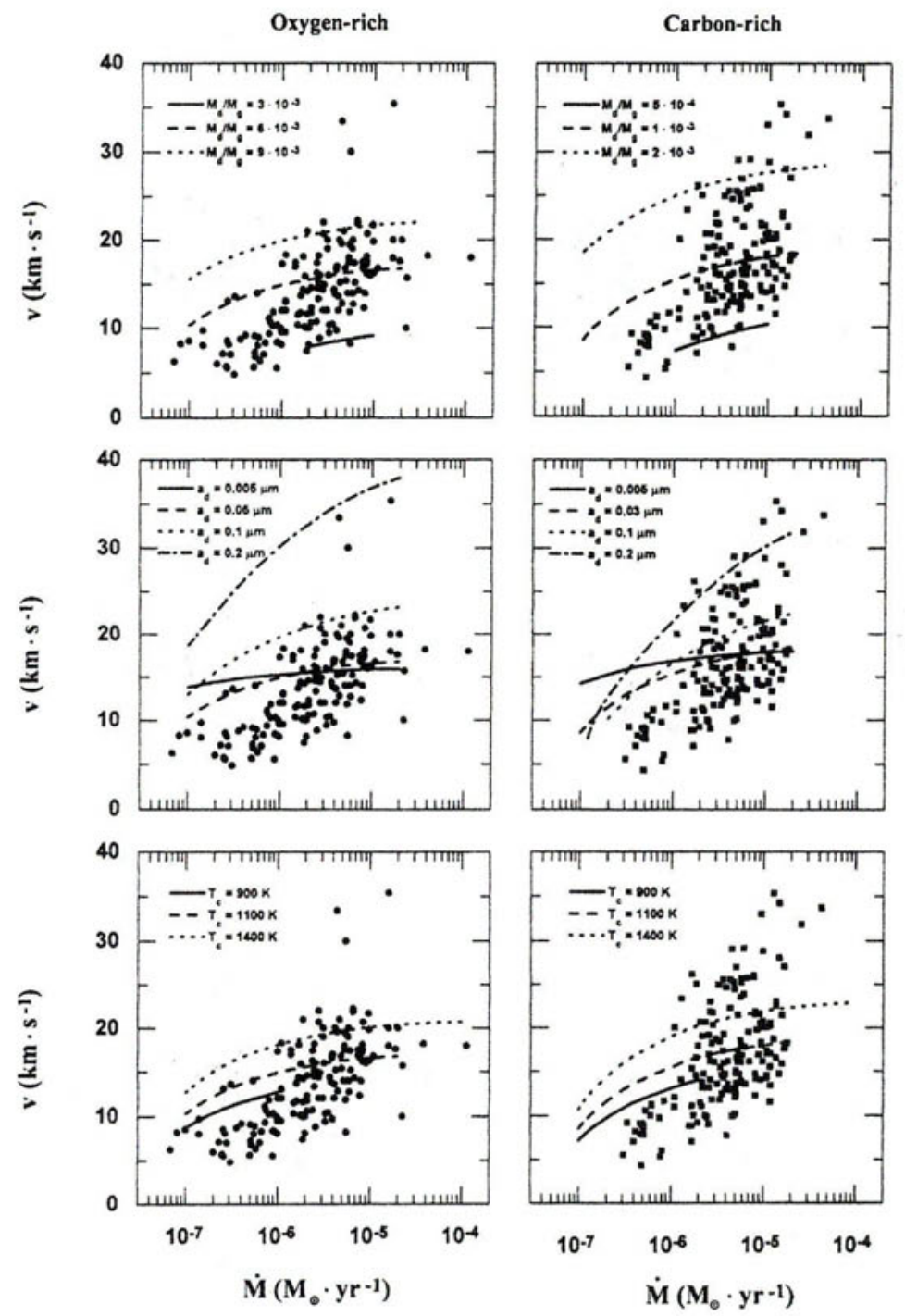

Fig. 2. Terminal outflow velocity versus mass-loss rate for the calculated models and individual stars. All model parameters which are not varied are those of the standard model (Table 1). Curves present model calculations for different dust-to-gas ratios (upper raw), grain radii (middle raw) and dust condensation temperatures (bottom raw). Individual observations are plotted as dots (oxygen-rich stars, left panels) and squares (carbon-rich stars, right panels). 

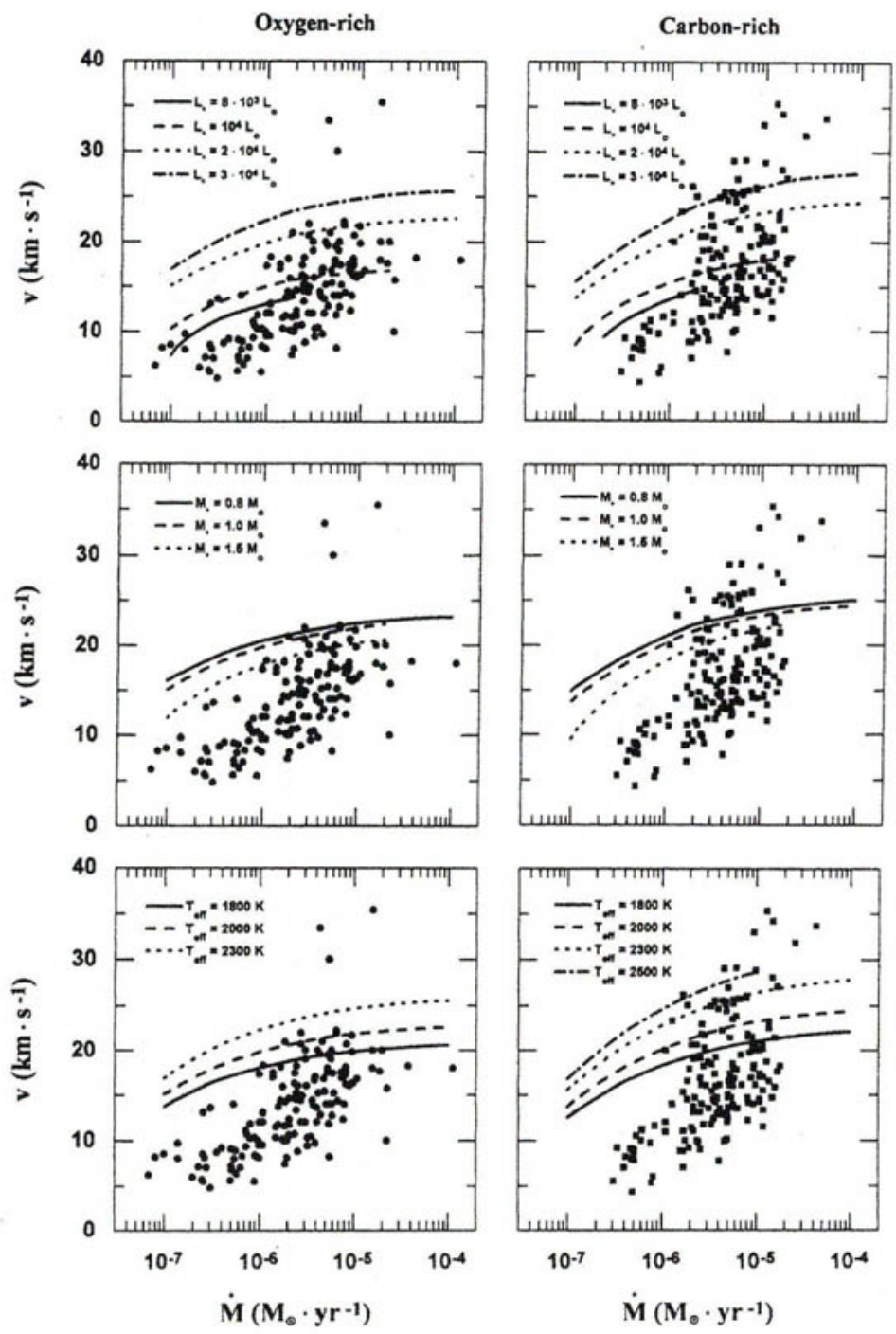

Fig. 3. Terminal outflow velocity versus mass-loss rate for the calculated models and individual stars. All model parameters which are not varied are those of the standard model (Table 1), except if stated otherwise. Curves present model calculations for different stellar luminosities (upper raw), masses (middle raw; $L_{\star}=2 \times 10^{4} L_{\odot}$ ) and effective temperatures (bottom raw; $L_{\star}=2 \times 10^{4} L_{\odot}$ ). Individual observations are plotted as dots (oxygen-rich stars, left panels) and squares (carbon-rich stars, right panels). 
dependence of $v_{\exp }$ on $a_{\mathrm{d}}$ is a consequence of nonlinear behavior of $Q_{\mathrm{pr}}$ with varying $a_{\mathrm{d}}$ (e.g., NE).

The position of the dust-forming region in the outflow may also play a role in determining terminal gas outflow velocities. Our calculations are based on the assumption that dust grains are necessarily formed, whatever the conditions in the dust forming region are. In reality, the dust condensation process is only possible when favorable physical and kinetic conditions are attained in the outflow. Our aim, however, was to investigate, independently of the particular dust formation pathway involved, to what extent higher or lower $T_{\mathrm{c}}$ may influence the resulting $v_{\exp }$. The influence of different $T_{\mathrm{c}}$ on the terminal gas outflow velocities is seen in Fig. 2. As the dust condensation point moves closer to the stellar surface (in case of higher $T_{\mathrm{c}}$ ), the flow is accelerated more effectively because of the resulting higher wind density (and hence the more effective momentum transfer between dust and gas). The opposite holds if the dust condensation point moves outward, while at a certain low $T_{\mathrm{c}}$ mass-loss ceases at all. It should be stressed, that in this case the mass-loss process stops because of the dynamical considerations alone (the momentum transfer is not effective enough to drive the outflow).

Hence, there is an indication that the terminal gas outflow velocities of the winds driven by the radiation pressure on dust grains are not only dependent on the amount and size of the dust grains formed, but also on the position of the dust forming region in the outflow. Although the influence of the dust condensation temperatures on the quantitative changes in $v_{\exp }$ is not very significant, the important fact is that the mass-loss process driven by the radiation pressure on dust may cease at all if the grains are to form at large distances from the stellar surface $\left(T_{\mathrm{c}}<800 \mathrm{~K}\right)$.

Different stellar luminosities can be also responsible for a quite large spread in terminal outflow velocities (Fig. 3). However, we have found that the mass-loss process driven by the radiation pressure on dust grains is possible only in stars of high luminosity $\left(L_{\star}>\right.$ $\left.7 \times 10^{3} L_{\odot}\right)$, regardless of the selected model input parameters. This agrees well with recent theoretical results of Fleischer et al. (1995) and Hofner et al. (1995), who have found that the wind, initiated by the instability due to the so-called exterior $\kappa$-mechanism caused by dust formation, can drive mass outflows only in cool and luminous objects. 
Terminal gas outflow velocities show only a slight dependence on stellar masses and effective temperatures (Fig. 3). We have found that dust driven outflows may occur in stars with masses up to 2 $3 M_{\odot}$ and effective temperatures up to $2700 \mathrm{~K}$, if a suitable combination of the remaining stellar parameters (such as luminosities, dust-to-gas ratios etc.) is selected. Typical masses and effective temperatures, however, are slightly lower (Fig. 3). The spread in stellar masses and effective temperatures cannot account for the whole observed range of outflow velocities and mass-loss rates.

Finally, our results confirm that there is a fairly good agreement between the observed and theoretically predicted terminal outflow velocities of the winds from evolved stars. As was indicated by Dominik et al. (1990), this may suggest that radiation pressure on dust grains may be dominating hydrodynamics in the outer regions of mass outflows from evolved stars. This may imply that stationary spherically symmetrical wind modeling might still be applicable at least as a first-order approximation to investigate certain dynamical properties of circumstellar outflows, especially at large distances from the stellar surface.

\section{CONCLUSIONS}

A comparison between theoretically predicted and observed $v_{\exp }$ indicates, that different dust-to-gas ratios $\dot{M}_{\mathrm{d}} / \dot{M}_{\mathrm{g}}$ may account for a wide range of the observed terminal outflow velocities and massloss rates for both oxygen-rich and carbon-rich stars on the AGB. Terminal gas outflow velocity may also be influenced significantly by stellar luminosity, though only marginally by stellar mass, effective temperature, average size of the dust grains and the position of the dust-forming region in the outflow.

Although the influence of dust condensation temperatures on the numerical values of $v_{\text {exp }}$ is not very significant, an important fact is that the mass-loss process driven by radiation pressure on dust may cease completely if the grains are to form at large distances from the stellar surface $\left(T_{\mathrm{c}}<800 \mathrm{~K}\right)$. It has to be stressed, that in this case the mass-loss process stops because of dynamical considerations alone (the momentum transfer is not effective enough to drive the outflow).

It is evident from the model calculations that radiation pressure on dust grains may drive the outflows only in cool $\left(T_{\text {eff }}<2700 \mathrm{~K}\right)$, 
luminous $\left(L_{\star}>7 \times 10^{3} L_{\odot}\right)$ and low mass $\left(M_{\star}<3 M_{\odot}\right)$ stars. Calculations also indicate that radiation pressure on dust grains generally cannot produce slow winds $\left(v_{\exp }<10 \mathrm{~km} / \mathrm{s}\right)$ at the low mass-loss rates $\left(\dot{M}_{\mathrm{g}}<10^{-6} M_{\odot} / \mathrm{yr}\right)$. All these facts suggest that radiation pressure on dust grains alone may operate only in a minor fraction of AGB stars, and that generally it has to be supported by some additional mechanisms (such as stellar pulsations).

ACKNOWLEDGMENTS. I am indebted to V. Vansevičius for numerous and very productive discussions on the manuscript. I would also like to thank S. Raudeliūnas for providing me with the Planck mean radiation pressure efficiency factors for silicate and amorphous carbon grains prior to publication and J. Mitton \& W. Buning for their help with computing resources. The Postdoctoral Research Fellowship with the Japan Society for the Promotion of Science is acknowledged.

\section{REFERENCES}

Allain T., Sedlmayr E., Leach S. 1997, A\&A, 323, 163

Bagnulo S., Doyle J. G., Griffin I. P. 1995, A\&A, 301, 501

Beck H.K.B., Gail H.P., Henkel R., Sedlmayr E. 1992, A\&A, 265, 626

Berruyer N., Frisch H. 1983, A\&A, 126, 269

Blocker T. 1995, A\&A, 297, 727

Bowen G. H. 1988, ApJ 329, 299

Cadwell B.J., Wang H., Feigelson E. D., Frenklach M. 1994, ApJ, 429, 285

Cherchneff I., Barker J.R., Tielens A. G. G. M. 1991, ApJ, 377, 541

Cherchneff I., Barker J.R., Tielens A. G. G. M. 1992, ApJ, 401, 269

Dominik C., Gail H.-P., Sedlmayr E. 1989, A\&A, 223, 227

Dominik C., Gail H.-P., Sedlmayr E., Winters J. M. 1990, A\&A, 240, 365

Dominik C., Sedlmayr E., Gail H.-P. 1993, A\&A, 277, 578

Draine B. T. 1985, ApJS, 57, 587

Egan M.P., Leung C. M. 1995, ApJ, 444, 251

Fleischer A.J., Gauger A., Sedlmayr E. 1992, A\&A, 266, 321

Fleischer A. J., Gauger A., Sedlmayr E. 1995, A\&A, 297, 543

Gail H.-P. 1992 in Instabilities in Evolved Super- and Hypergiants, eds. C. de Jager \& H. Nieuwenhuizen, Amsterdam

Gail H.-P., Sedlmayr E. 1985, A\&A, 148, 183

Gail H.-P., Sedlmayr E. 1986, A\&A, 166, 225

Gail H.-P., Sedlmayr E. 1987, A\&A, 171, 197 
Gail H.-P., Keller R., Sedlmayr E. 1984, A\&A, 133, 320

Gilman R. 1974, ApJS, 28, 397

Groenewegen M. A. T. 1997, A\&A, 317, 503

Groenwegen M.A. T., van de Hoek L. B., de Jong T. 1995, A\&A, 293, 381

Habing H.J. 1996, A\&AR, 7, 97

Habing H.J., Tignon J., Tielens A. G. G. M. 1994, A\&A, 286, 523 (HTT)

Hartquist T. W., Havnes O. 1994, A\&SS, 218, 23

Helling C., Jorgensen U. G., Plez B., Johnson H. R. 1996, A\&A, 315, 194

Hofner S., Feuchtinger M., Dorfi E. A. 1995, A\&A, 297, 815

Ivezic Z., Elitzur M. 1995, ApJ, 445, 415

Ivezic Z., Elitzur M. 1996, MNRAS, 279, 1019

Jorgensen U. G. 1994, A\&A, 284, 179

Jorgensen U. G., Johnson H.R. 1992, A\&A, 265, 168

Jorgensen U. G., Johnson H. R., Nordlund A. 1992, A\&A, 261, 263

Jura M. 1994, ApJ, 434, 713

Kohler T. M., Gail H.-P., Sedlmayr E. 1997, A\&A, 320, 553

Kruger D., Gauger A., Sedlmayr E. 1994, A\&A, 290, 573 (KGS)

Kruger D., Woitke P., Sedlmayr E. 1995, A\&AS, 113, 593

Kruger D., Sedlmayr E. 1997, A\&A, 321, 557 (KS)

Loup C., Forveille T., Omont A., Paul J.F. 1993, A\&AS, 99, 291

MacGregor K. B., Stencel R.E. 1992, ApJ, 397, 644

Marigo P., Bressan A., Chiosi C., 1996, A\&A 313, 545

Martin P. G., Rogers C. 1987, ApJ, 322, 374

Mastrodemos N., Morris M., Castor J. 1996, ApJ, 468, 851

Netzer N., Elitzur M. 1993, ApJ, 410, 701 (NE)

Olofsson H., Eriksson K., Gustafsson B., Carlstrom U. 1993a, ApJS, 87, 267

Olofsson H., Eriksson K., Gustafsson B., Carlstrom U. 1993b, ApJS, 87, 305

Papoular R., Pegourie B. 1986, A\&A, 156, 199

Plez B. 1992, A\&AS, 94, 527

Raudeliūnas S. 1997, private communication

Rouleau F., Martin P. G. 1991, ApJ 377, 526

Rowan-Robinson M., Harris S. 1982, MNRAS, 200, 197

Rowan-Robinson M., Harris S. 1983, MNRAS, 202, 767

Salaris M., Cassisi S. 1996, A\&A, 305, 858

Seab C. B., Snow T.P. 1989, ApJ, 347, 479

Steffen M., Szczerba R., Menshchikov A., Schoenberner D. 1997, A\&AS, 126,39

Tielens A.G. G. M. 1990, in From Miras to PN: which Path for Stellar

Evolution?, ed. M. O. Mennessier, Editions Frontieres

Weidemann V. 1987, A\&A, 188, 74 